\title{
Advantage of Platelet-rich Fibrin and Lateral Position Pedicle Flap Technique for the Treatment of Gingival Recession
}

\author{
Abhishek Gautam
}

\begin{abstract}
Aim: The main aim of the study is to analyze the adequacy of platelet-rich fibrin and lateral position pedicle flap method over laterally sliding flap technique to cure gingival recession.

Background: Nowadays, second-generation platelet concentrate having autologous platelet-rich fibrin (PRF) gel along with growth factors that have cicatricle properties is being used for exposed root in dentistry. This technique is a recent invention to treat exposed roots with adequacy on various existing methods. Dentists have found numerous clinical challenges in treatment of gingival recession by laterally sliding flap technique that is being used commonly to cover isolated, denuded roots at vestibular depth. Laterally sliding flap technique has been modified due to undesirable results on the donor teeth. Due to limitations of the abovementioned methods, growth factors are found suitable in combination with surgical methods.

Case description: After using platelet-rich fibrin and lateral position pedicle flap method along with autologous suspension of growth factor over laterally sliding flap technique, the patients were found stable with about $80-85 \%$ root coverage as well as satisfactory healing in gingival tissues in both donor and recipient site with no inflammation.

Conclusion: This technique has very satisfactory outcome as the patients were satisfied with case resolution.

Clinical significance: Second-generation platelet concentrate having autologous PRF have the properties to promote tissue repair and regeneration. This method does not have second surgical site because the donor tissue is taken from the same surgical site.

Keywords: Gingival recession, Lateral pedicle, Platelet-rich fibrin.

International Journal of Experimental Dental Science (2020): 10.5005/jp-journals-10029-1204
\end{abstract}

\section{INTRODUCTION}

Gingival recession is the apical migration of marginal gingiva that displaced from cementoenamel junction. ${ }^{1}$ It may affect all surfaces of the tooth in localized area due to behavioral habits that may swell the gingival connective tissues which results in shrinkage and recession of the gingival tissues. ${ }^{2}$ This shrinkage may lead to root hypersensitivity and root caries. ${ }^{3}$ In mid of 20th century, this disease was treated by free autogenous grafts, pedicle grafts including rotational flaps, coronally advanced flaps (CAFs), and semilunar flaps methods. Later, combination of autogenous or allograft as well as guided tissue regeneration membranes techniques were invented to cure such disease. ${ }^{4}$ Dentists have found numerous clinical challenges in treatment of gingival recession by laterally sliding flap technique which is the commonly used technique to cover isolated, denuded roots at vestibular depth. ${ }^{5}$ Laterally positioned pedicle flap-revised technique introduced by Ruben et al. ${ }^{6}$ have been found more suitable than laterally sliding flap technique that was described by Harvey ${ }^{7}$ due to undesirable results on the donor teeth. This method is quite useful in connective tissue-cementum fixation of the flap that prohibits the postoperative complication of bone resorption by preserving the periosteal protection and blood supply to the septum. ${ }^{8}$ Nowadays, second-generation platelet concentrate with autologous platelet-rich fibrin gel (PRF) along with growth factors that have cicatricle properties is being used for exposed root in dentistry. ${ }^{9}$ The main aim of the study is to analyze the adequacy of PRF and lateral position pedicle flap method over laterally sliding flap technique to cure gingival recession.

\section{Case Description}

A patient with the problem of receding gums and sensitivity of lower gums came to Department of Dentistry, Anugrah Narayan
Department of Dentistry, Anugrah Narayan Magadh Medical College and Hospital, Gaya, Bihar, India

Corresponding Author: Abhishek Gautam, Department of Dentistry, Anugrah Narayan Magadh Medical College and Hospital, Gaya, Bihar, India, Phone: +91 7870106356, e-mail: dr.abhi90@gmail.com

How to cite this article: Gautam A. Advantage of Platelet-rich Fibrin and Lateral Position Pedicle Flap Technique for the Treatment of Gingival Recession. Int J Experiment Dent Sci 2020;9(1):13-15.

Source of support: Nil

Conflict of interest: None

Magadh Medical College and Hospital, Gaya. Clinical diagnosis reflects Miller class-I recession defect at 31. Buccal aspect of the tooth shows a loss of $6 \mathrm{~mm}$ that reflects the distance between the cementoenamel junction and gingival margin of $4 \mathrm{~mm}$ and the distance between the gingival margin and the base of the pocket of $2 \mathrm{~mm}$ above mucogingival junction (Fig. 1).

\section{Treatment Plan}

Laterally positioned flap with PRF membrane was speculated to cover gingival recession.

\section{Presurgical Therapy}

The patient was investigated regularly that included scaling, root planing, and oral hygiene. Patient was prescribed modified Stallman's brushing technique. Parameters assessed included the following. UNC - 15 periodontal probes were assessed at baseline and at 6 months after surgical procedure. Clinical recession depth (RD), location between cementoenamel junction to the most apical point of gingival margin and the bottom of the gingival sulcus, 


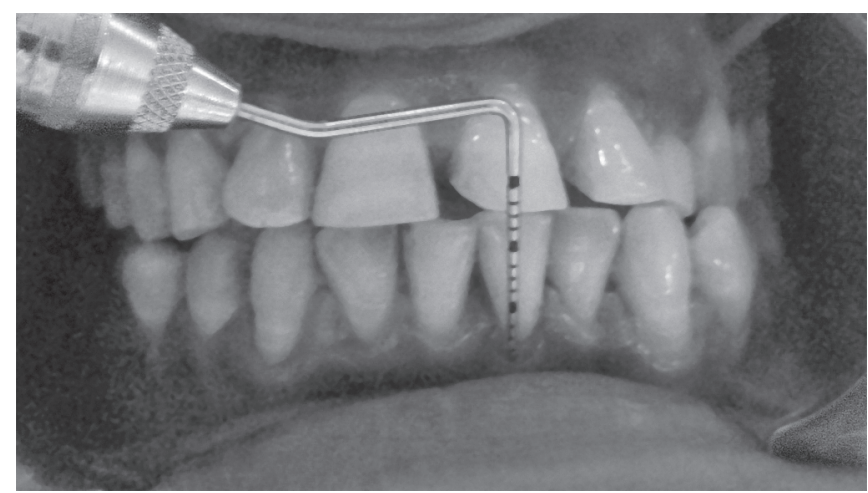

Fig. 1: Preoperative view

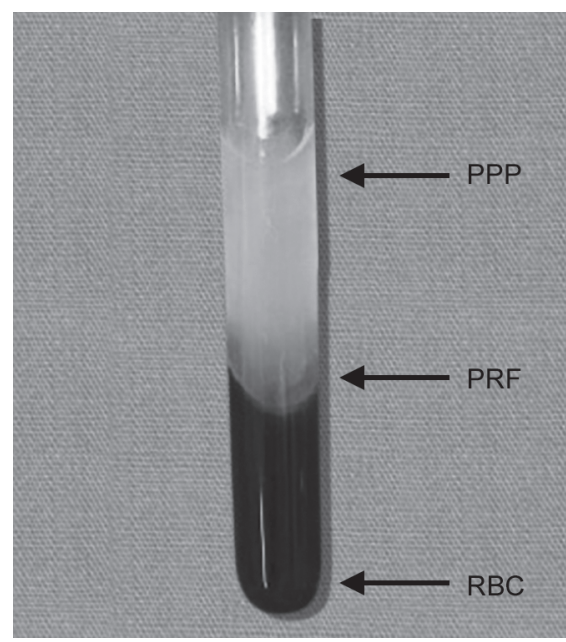

Fig. 3: Blood sample separated in test tube

was analyzed. Like clinical recession depth, clinical attachment level (CAL) was also noticed from cement-enamel junction to the bottom of the pocket.

\section{Surgical Procedure}

Laterally positioned pedicle flap with PRF technique was used. The operative site was anesthetized using $2 \%$ xylocaine hydrochloride with adrenaline; later the collar tissue was removed by two vertical incisions at apical to base of the recession. Scaling and root planning were performed at the donor site by a vertical incision from gingival margin to oral mucosa by 15-number blade through the periosteum at its apical base (Fig. 2)

\section{Preparation of PRF}

Blood ( $5 \mathrm{~mL}$ ) was extracted and kept in $10 \mathrm{~mL}$ test tube in the absence of anticoagulant and centrifuged at 3,000 rpm for 10 minutes. This forms three layers in centrifuge tube: acellular plateletpoor plasma stays in upper layer, PRF clot is observed in the middle layer, and red blood cell has been observed in lower layer (Fig. 3). Fibrin clot was crushed to obtain PRF membrane (Fig. 4) which was kept in denuded roots and stabilized (Fig. 5). The flap fully wraps the membrane using sling sutures (Fig. 6). Periodontal dressing was done on the wounded site (Fig. 7).

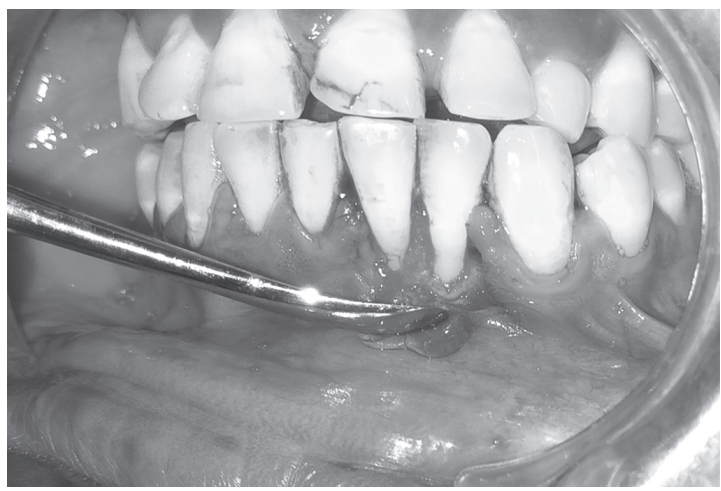

Fig. 2: Combined partial- and full-thickness flap elevated

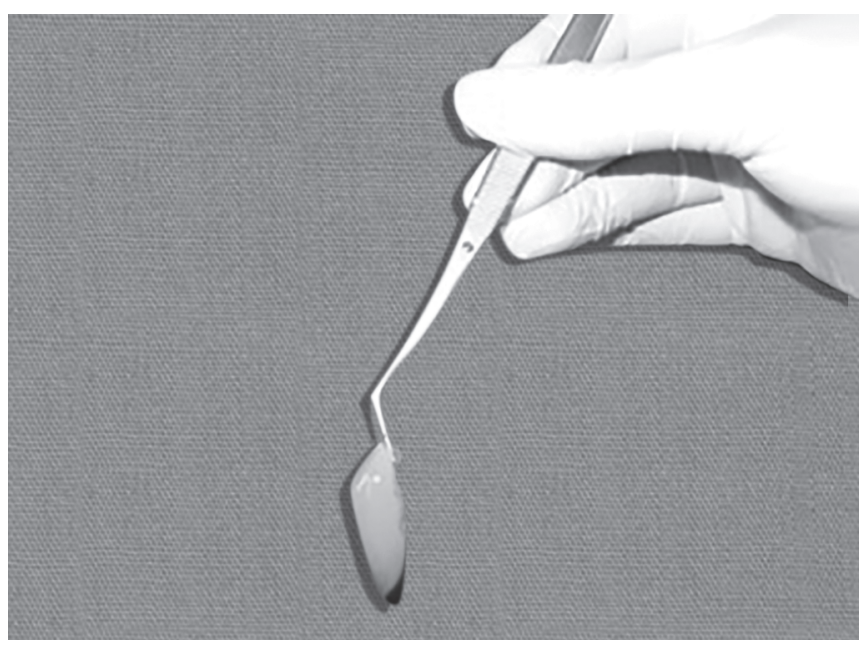

Fig. 4: Squeezed PRF

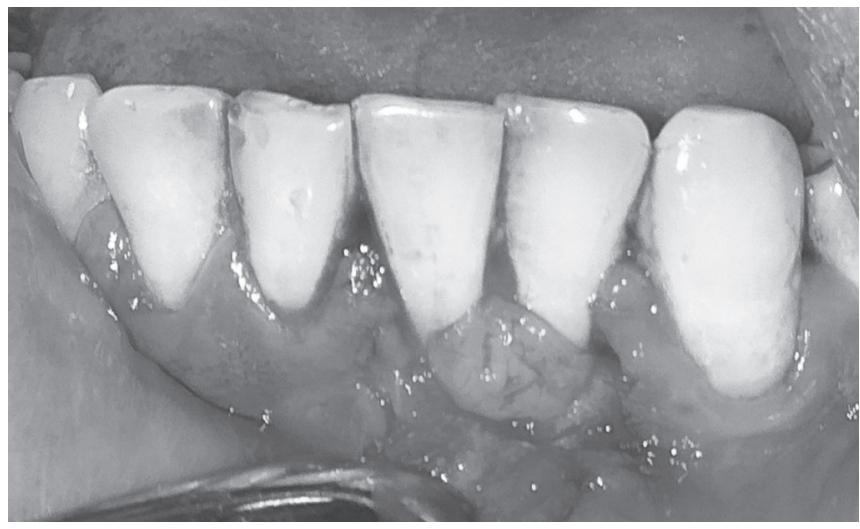

Fig. 5: PRF placed

\section{Postoperative Care}

The patient was advised to rinse mouth by $0.12 \%$ chlorhexidine digluconate for 2 weeks which maintains hygiene. Amoxicillin $500 \mathrm{mg}$, ibuprofen $400 \mathrm{mg}$ thrice a day, and vitamin B-complex once a day were prescribed for 5 days. Dressing as well as sutures was stopped after 12 days, and follow-up at 6 months showed no complication with satisfactory healing. 


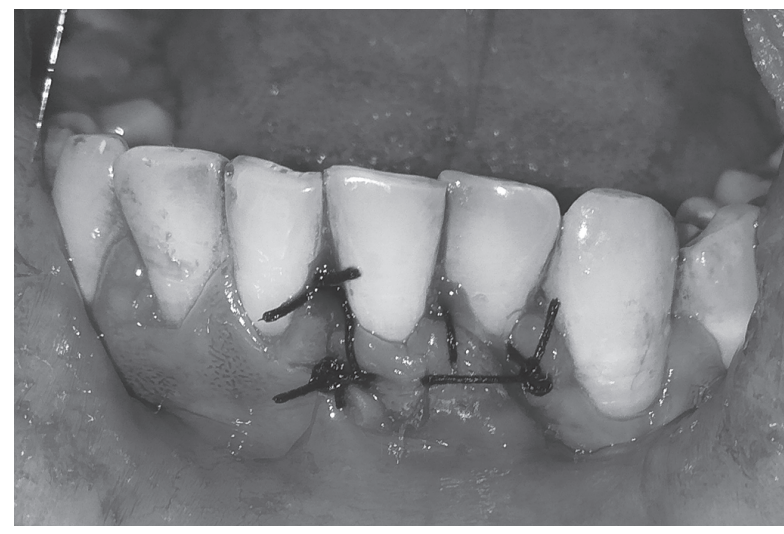

Fig. 6: Sling suture placed

\section{Results}

After 6 months of treatment, the patient showed almost $80 \%$ improvements at treated site with $5-\mathrm{mm}$ increase in CAL, 4-mm decrease in recession, and 1-mm lessening in probing depth with admirable contour and color. No inflammation was observed on final evaluation in the gingival tissues of both the donor and recipient site.

\section{Discussion}

Numerous surgical methods have been described to treat gingival recession and one of the successful methods is root coverage in periodontics. Laterally positioned pedicle flap revised technique introduced by Ruben et al. ${ }^{6}$ have been found more suitable than laterally sliding flap technique that was described by Harvey ${ }^{7}$ due to undesirable results on the donor teeth. This method is quite useful in connective tissue-cementum fixation of the flap that prohibits the postoperative complication of bone resorption by preserving the periosteal protection and blood supply to the septum. ${ }^{8}$ These lesions endow with osteogenic, fibrogenic, vascular proliferation, cementogenic, and neural trophic activity that exhibits significant role in healing of the periodontal surgical wound, which may avoid the exposure of the bone at the donor site. ${ }^{9}$ Root coverage procedures are quite useful in healing junctional epithelium as well as regeneration along apical portion of the lesion. ${ }^{10}$ Polypeptide growth factors and platelet-rich plasma (PRP) have been used for periodontal regeneration. PRP is kept in anticoagulant that may inhibit platelet activation and degranulation. After centrifugation, PRP is mixed with bovine thrombin and calcium chloride at the time of application. ${ }^{11}$ However, platelet-rich fibrin has a number of advantages over PRP that remove the jeopardy associated with the use of bovine thrombin although both have different protocols. ${ }^{12}$ It also provides a significant postoperative protection of the surgical site. ${ }^{13}$ In PRF preparation, care should be taken in collection of blood and centrifugation in the absence of anticoagulant because blood starts getting coagulated as soon as in contact with glass surface. Large sample size is required to emphasize PRF's impact on softtissue reconstruction in periodontal therapy along with laterally positioned pedicle flap-revised technique.

\section{Conclusion}

It may be concluded that the adequacy of platelet-rich fibrin and lateral position pedicle flap method over laterally sliding flap technique to cure gingival recession defects is quite useful when

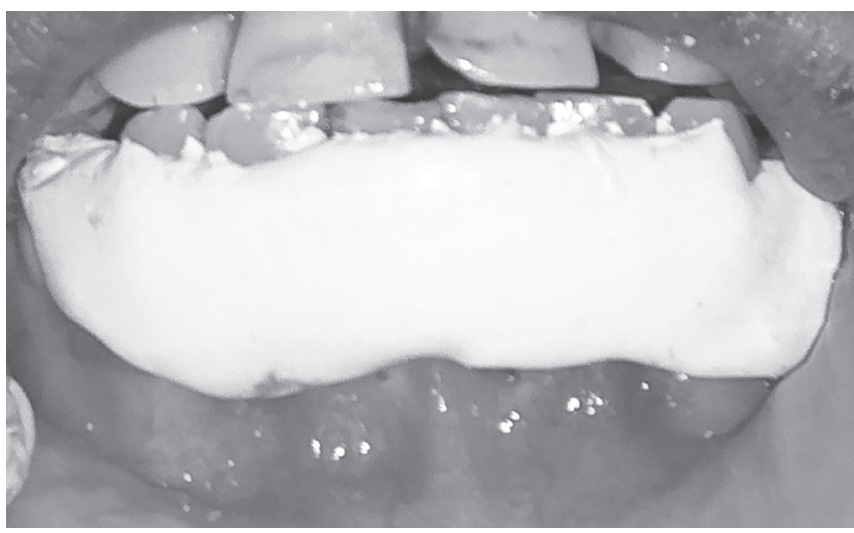

Fig. 7: Coe pack placed

compared to restore the functional properties of labial gingiva of teeth.

\section{References}

1. Jan LW, Giovanni Z. Increased gingival dimensions. A significant factor for successful outcome of root coverage procedures?: a 2-year prospective clinical study. J Clin Periodontol 1996;23(8):770-777. DOI: 10.1111/j.1600-051X.1996.tb00608.x.

2. Allen $\mathrm{E}$, Irwin $\mathrm{C}$, Ziada $\mathrm{H}$, et al. Periodontics: The management of gingival recession. Dent Update 2007;34(9):534-536. DOI: 10.12968/ denu.2007.34.9.534, 538-40, 542.

3. Moawia MK, Robert EC. The etiology and prevalence of gingival recession. J Am Dent Assoc 2003;134(2):220-225. DOI: 10.14219/jada. archive.2003.0137.

4. Anilkumar K, Geetha A, Umasudhakar, et al. Platelet-rich-fibrin: a novel root coverage approach. J Indian Soc Periodontol 2009;13(1):50-54. DOI: 10.4103/0972-124X.51897.

5. Carranza S. Clinical Periodontology Newman MG, Takei HH, Klokkevold PR, et al., ed. 10th ed., ST. Louis, Missouri: Saunders Publication; 2006. p. 1016.

6. Ruben MP, Goldmon HM, Janson W. Biological considerations fundamental to successful employment of laterally repositioned pedicle flaps and free autogenous gingival grafts in periodontal therapy Stahl S, ed., ch. 9 Periodontal Surgery. Springfield: Illinois; 1976.

7. Harvey PM. Surgical reconstruction of the gingiva. Part II. Procedures. N Z Dent J 1970;66(303):42-52.

8. De waal H, Kon S, Ruben MP. The laterally positioned flap. Dent Clin North Am 1988;32(2):267-285.

9. Dohan DM, Choukroun J, Diss A, et al. Platelet-rich fibrin (PRF): a second generation platelet concentrate. Part I: technological concepts and evolution. Oral Surg Oral Med Oral Pathol Oral Radiol Endod 2006;101(3):e37-e44. DOI: 10.1016/j.tripleo.2005.07.008.

10. Grupe $H E$, Warren RE. JR repair of gingival defects by a sliding flap operation. J Periodontol 1956;27(2):92-95. DOI: 10.1902/ jop.1956.27.2.92.

11. Aroca S, Keglevich T, Barbieri B, et al. Clinical evaluation of a modified coronally advanced flap alone or in combination with a platelet-rich fibrin membrane for the treatment of adjacent multiple gingival recessions: a 6-month study. J Periodontol 2009;80(2):244-251. DOI: 10.1902/jop.2009.080253.

12. Toffler M, Toscano M, Holtzclaw D, et al. Introducing Choukroun's platelet rich fibrin (PRF) to the reconstructive surgery milieu. JIACD 2009;1:21-32.

13. Choukroun J, Diss A, Simonpieri A, et al. Platelet rich fibrin (PRF): a second generation platelet concentrate. Part V: histologic evaluations of PRF effects on bone allograft maturation in sinus lift. Oral Surg Oral Med Oral Pathol Oral Endod 2006;101(3):299-303. DOI: 10.1016/j. tripleo.2005.07.012. 